\title{
X-RAY SOURCES AS TRACERS OF THE LARGE-SCALE STRUCTURE IN THE UNIVERSE
}

\author{
X. Barcons ${ }^{1}$, F.J. Carrera ${ }^{2,1}$, M.T. Ceballos ${ }^{1}$, S. Mateos ${ }^{1,3}$ \\ 1) Instituto de Física de Cantabria (CSIC-UC), 39005 Santander, Spain \\ 2) Mullard Space Science Laboratory, University College London, UK \\ 3) Departamento de Física Moderna, Universidad de Cantabria, 39005 Santander, Spain
}

\begin{abstract}
We review the current status of studies of large-scale structure in the X-ray Universe. After motivating the use X-rays for cosmological purposes, we discuss the various approaches used on different angular scales including X-ray background multipoles, cross-correlations of the X-ray background with galaxy catalogues, clustering of X-ray selected sources and small-scale fluctuations and anisotropies in the X-ray background. We discuss the implications of the above studies for the bias parameter of X-ray sources, which is likely to be moderate for X-ray selected AGN and the X-ray background $\left(b_{X} \sim 1-2\right)$. We finally outline how all-sky X-ray maps at hard X-rays and medium surveys with large sky coverage could provide important tests for the cosmological models.
\end{abstract}

KEYWORDS: Large-scale structure of Universe; cosmology; galaxies: active,clustering.

\section{INTRODUCTION}

In the current cosmological picture, galaxies, clusters and large-scale structures have grown from initial small perturbations in the density of the Universe via gravitational collapse. Cosmological models are required to meet two basic observational constraints: on the one hand the Universe at $z \sim 1500$ was very smooth, as the cosmic microwave background (CMB) is seen to have anisotropies of amplitude $\sim 10^{-5}$; on the other hand local mass inhomogeneities measured through the distribution of galaxies exhibit fluctuations of the order $\sim 1$ on scales $\sim 10 \mathrm{Mpc}$. Different cosmologies, however, predict highly discrepant ways in which structures on different scales grow up to the current state from the CMB initial conditions. The largest discrepancies occur at redshifts $z \sim 1-5$ which is when galaxies began to collapse and to form stars. Accessing these intermediate redshifts will provide crucial tests for the cosmological models.

The isotropy of the cosmic X-ray background (XRB) on large angular scales ( $\frac{\Delta I}{I}$ less than a few $\%$ on scales of degrees and larger) suggests that most of the $\mathrm{X}$ ray photons we receive from the Universe must have been originated in the distant Universe. Surveys at different depths carried out with ROSAT have revealed that $50-70 \%$ of the (soft) XRB is resolved into point sources, mostly Active Galactic 
Nuclei (AGN) of different classes. Although there are still some discrepancies in the determination of the $\mathrm{X}$-ray luminosity function and its redshift evolution, there is no doubt that most of the XRB originates at redshift $z>1$. Boyle et al (1994) and Page et al (1996) who find their samples of X-ray selected AGN consistent with pure luminosity evolution models, predict a peak in the X-ray volume emissivity around $z \sim 1.5-2$. Miyaji et al (1998) instead find better consistency with luminosity dependent density evolution, in which case the X-ray volume emissivity in AGN more luminous than $10^{44.5} \mathrm{erg} \mathrm{s}^{-1}$ (which for the broken power-law shape of the luminosity function account for most of the X-rays emitted by AGN) rises steeply from $z=0$ to $z=1-2$ with no evidence for a decline at higher redshifts. In both cases it is clear that soft X-ray emission from the extragalactic sky comes mostly from redshifts $z=1-2$ or larger, in a situation very similar to the star formation in the Universe (Madau et al 1996, Boyle \& Terlevich 1998). Studying the X-ray Universe is then likely to provide a major handle to understand the evolution of the Universe at intermediate redshifts and therefore it is an issue of prime cosmological relevance.

There are other reasons to prefer X-rays to carry out cosmological studies. On the one hand the high-latitude X-ray sky is 'clean', at least at photon energies above $2 \mathrm{keV}$, galactic absorption has negligible effects and the contribution of the Galaxy to the XRB is less than a few \% (Iwan et al 1982). A further reason is the small content in stars of high galactic latitude surveys, ranging from $25 \%$ at bright fluxes down to probably less than $10 \%$ at the faintest fluxes.

In this paper we review the current status of studies of the large-scale structure of the Universe, which up to now has produced relevant but certainly not spectacular results. The two main questions that we address are:

- Do X-ray sources (and the XRB) trace mass in the Universe and what is their bias parameter?

- What are the best observational approaches to obtain information on the large-scale structure of the Universe at intermediate redshifts with X-rays?

Except when otherwise stated we use $H_{0}=100 h \mathrm{~km} \mathrm{~s}^{-1} \mathrm{Mpc}^{-1}, q_{0}=0.5$ and $\Lambda=0$.

\section{THE X-RAY SKY ON THE LARGEST SCALES}

The distribution of the XRB fluctuations on the largest scales and their link to inhomogeneities in the distribution of matter has been an active field of research for many years. The observational resources have been mostly limited to the HEAO1 A2 experiment which scanned the sky with a resolution of $3^{\circ} \times 1.5^{\circ}$ at photon energies 2-60 keV. 


\begin{tabular}{|l|c|c|c|c|l|}
\hline & $l(d e g)$ & $b(d e g)$ & Err $(d e g)$ & XRB ampl (\%) & Ref \\
\hline CMB & 264 & 48 & & 0.15 & F96 \\
AGN & 318 & 38 & 30 & & MB90 \\
Clusters & 260 & 5 & 15 & & PK98 \\
Soft XRB & 288 & 25 & 19 & 1.7 & PG99 \\
Hard XRB & 338 & 47 & 25 & 0.11 & S99 \\
\hline
\end{tabular}

TABLE 1. Dipoles of X-ray source populations and the XRB. References are Fixsen et al (1996); MB80: Miyaji \& Boldt (1980); PK98: Plionis \& Kolokotronis (1998); PG99: Plionis \& Georgantopoulos (1999); S99: Scharf et al (1999).

\subsection{The dipole of X-ray sources}

Since the Galaxy is moving with respect to the frame where the CMB would be isotropic towards $l=264^{\circ}, b=48^{\circ}$, there must be an overdensity of sources which are pulling us towards that direction. The distribution of X-ray sources in the sky should therefore exhibit an approximate large-scale dipolar distribution pointing towards the same direction.

Using the AGNs in the Piccinotti et al (1982) flux-limited sample of X-ray sources (2-10 keV flux limit $\sim 3 \times 10^{-11} \mathrm{erg} \mathrm{cm}^{-2} \mathrm{~s}^{-1}$ ), Miyaji \& Boldt (1990) and Miyaji (1994) found the dipole of these sources to point towards $l=318^{\circ}, b=$ $38^{\circ}$ with a large error circle $\left(\sim 30^{\circ}\right.$ radius $)$. The dipole appears to saturate at $50-100 h^{-1} \mathrm{Mpc}$ and is roughly aligned with the CMB dipole. Within the framework of linear theory, this allows the bias parameter of the X-ray selected AGN to be estimated, giving a somewhat large value $\left(b_{X} \Omega_{0}^{-0.6} \sim 3-6\right)$. Uncertainties come primarily from the indetermination of the redshift at which the dipole saturates.

Plionis \& Kolokotronis (1998) and Kolokotronis et al (1998) have measured the dipole of an X-ray flux-limited sample of galaxy clusters. This is again in rough alignment with the CMB dipole, but it appears to saturate at $\sim 160 h^{-1} \mathrm{Mpc}$. As expected in all popular scenarios where clusters arise in extreme peaks of the underlying dark-matter distribution, they exhibit a large bias parameter $\left(b_{X} \sim 4\right.$, see Table 4).

The fact that the dipoles of the two most numerous classes of extragalactic X-ray sources (AGNs and clusters) are roughly aligned with the CMB dipole is encouraging. We note, however, that all-sky deeper samples of these objects (particularly X-ray selected AGN) would enormously help in defining the distance at which the contribution to the dipole saturates and therefore in measuring the bias parameter.

\subsection{The dipole of the X-ray background}

There are two reasons why the XRB should show a dipole signal: our motion relative to the CMB rest frame (the so-called Compton-Getting effect) and the excess contribution of the sources that cause this motion in the same direction. The XRB 
dipole is expected to be aligned with the CMB dipole, but the amplitude should be larger than the Compton-Getting effect, allowing for the excess emissivity.

There are two basic problems in measuring the XRB dipole: one is the contribution of the Galaxy and the other one is the integrated nature of the XRB whereby confusion noise dominates on all angular scales. Warwick, Pye \& Fabian (1980) realized that even at photon energies $>2 \mathrm{keV}$ and galactic latitudes $|b|>20^{\circ}$ a residual galactic contribution $\sim 2-7 \%$ is present. Iwan et al (1982) modelled this galactic component in terms of a finite radius disk with thermal spectrum at $T \sim 9$ $\mathrm{keV}$. To emphasize how difficult is to obtain the extragalactic signal, the galactic contribution amounts to a few $\%$ at the galactic poles, while the effect it is being looked for is less than $1 \%$.

Attempts to look for singular enhancements of the XRB surface brightness include those by Warwick et al (1980), the Jahoda \& Mushotzky (1989) search for emissivity from the great attractor, the Mushotzky \& Jahoda (1992) search for XRB negative fluctuations towards the most prominent voids and the unsuccessful detection of X-ray emission from superclusters by Persic et al (1990).

By modelling out the Galaxy, Shafer (1983) and Shafer \& Fabian (1983) found a dipole signal significant at $\sim 2 \sigma$ level in the HEAO-1 A2 map. Most of the subsequent dipole refinements have used the same data with increasingly finer corrections for detector drifts and other unwanted effects. The latest one is by Scharf et al (1999), who excluded the galactic plane, the Magellanic clouds and also regions around the Piccinotti et al (1982) sources, which leaves less than $50 \%$ of the sky for the dipole analysis. Various methods are used to deal with the masked regions (including spherical harmonic reconstruction) and the results are shown in Table 1. The dipole signal is very clearly detected and its intensity appears larger than the Compton-Getting effect. The direction of this extra large-scale structure dipole caused by the fluctuations in the source density is only roughly aligned with the direction of our motion, and its amplitude is similar to the Compton-Getting effect as predicted by theory (Lahav, Piran \& Treyer 1997).

In an analysis of the ROSAT all-sky data $(0.9-2.4 \mathrm{keV})$, Plionis \& Georgantopoulos (1999) also find a dipole component. The Galaxy is modeled according to the Iwan et al (1982) model and they further exclude other regions associated with the Galaxy. The direction of the resulting dipole is in better agreement with the CMB dipole, but the amplitude is almost a factor of 10 larger than the ComptonGetting effect.

There are various reasons for the discrepancy between these measurements. First, an extra residual contribution from the Galaxy is likely to contaminate more strongly the ROSAT data than the HEAO-1 A2 data. This would explain why the ROSAT dipole points closer to the galactic plane and that its amplitude is larger. A second reason for the discrepancy is the fact that Scharf et al (1999) have excluded regions around the galaxy clusters present in the Piccinotti et al (1982) sample (which are known to have a very large bias parameter and represent $50 \%$ of the extragalactic sources in that sample) but Plionis \& Georgantopoulos (1999) have not. In fact these last authors note that the contribution from the Virgo cluster 
alone is of the order of $20 \%$ of the detected dipole. A good exercise which could give some insight on the level of the galactic contamination in the ROSAT data would be to exclude the clusters in the ROSAT analysis and not excise the Piccinotti et al (1982) sources from the analysis of the A2 data.

\subsection{Higher order multipoles of the X-ray background}

Lahav, Piran \& Treyer (1997) proposed the use of a multipole expansion of the angular variations of the XRB in order to measure the large-scale structure of the Universe. Under fairly general assumptions, the coefficients $a_{l m}$ of the harmonic expansion would be the sum of a large-scale structure term $a_{l m}^{(L L S)} \propto l^{-0.4}$ and a confusion noise term which is a function of the flux $S_{c u t}$ down to which sources have been excised from the maps for the multipole analysis $a_{l m}^{(N o i s e)} \propto S_{c u t}^{\gamma-1}$, where $\gamma$ is the slope of the integral source counts in the energy band used $\left(N(>S) \propto S^{-\gamma}\right)$.

Treyer et al (1998) performed this analysis on the HEAO-1 A2 all sky data by removing regions around the Piccinotti et al (1982) sample and the galactic plane. They find evidence for a growth of the spherical harmonic coefficients growing at low values of $l$ in a manner roughly consistent with the predictions. The significance of the signal is difficult to assess as the harmonic coefficients are not independent due to cross-talk between different orders introduced by the masking. Assuming a redshift dependent bias parameter for the X-ray sources parametrized as $b_{X}(z)=$ $b_{X}(0)+z\left[b_{X}(0)-1\right]$ (which assumes that all galaxies form at some past epoch, Fry 1996), they estimate a rather modest bias parameter $\left(1.0<b_{X}(0)<1.6\right)$. In their diagrams it is also seen that the dipole $(l=1)$ has an unusually large amplitude compared to higher harmonics.

The way to go is indeed to have precise measurements of the XRB intensity on large angular scales, but with the possibility of excluding sources down to the faintest possible levels. Treyer et al (1998) suggest that an all-sky map with XRB intensities measured with a $1 \%$ precision and with sources excised down to $3 \times$ $10^{-13} \mathrm{erg} \mathrm{cm}^{-2} \mathrm{~s}^{-1}$ (i.e., 100 times fainter than the Piccinotti et al catalogue) would be ideal for a spherical harmonic analysis.

\section{CROSS-CORRELATIONS OF GALAXY CATALOGUES WITH XRB INTEN- SITIES}

An alternative way that has been devised to look for structure in the X-ray sky is to cross-correlate the unresolved XRB intensity with catalogues of galaxies. The amplitude of the cross-correlation function $(\mathrm{CCF})$ between the X-ray intensity $I_{X R B}$ and the galaxy surface density $N_{g}, W_{X g}(\theta)=\left\langle I_{X R B} N_{g}\right\rangle_{\theta} /\left\langle I_{X R B}\right\rangle\left\langle N_{g}\right\rangle-1$ at zerolag $(\theta=0)$ provides an approximate measurement of the fraction of the XRB arising either in the catalogued galaxies or in sources clustered with them within a scale of the beam with which X-ray observations have been obtained (Lahav et al 1993).

Positive signals have been found for $W_{X g}$, typically of the order of $1 \%$ when the galaxies are optically or infrared selected, and up to $>10 \%$ when active galaxies 


\begin{tabular}{|l|l|c|l|}
\hline XRB data & Galaxy catalogue & $W_{X G}(0)$ & Ref \\
\hline HEAO-1 A2 & IRAS 2Jy & $7 \times 10^{-3}$ & M94 \\
GINGA NGP & IRAS 0.7Jy & $1.4 \times 10^{-2}$ & C95 \\
GINGA NGP & UGC & $1.1 \times 10^{-2}$ & C95 \\
HEAO-1 A2 & IRAS 12 $\mu$ all & $<9.6 \times 10^{-3}$ & B95 \\
HEAO-1 A2 & IRAS $12 \mu$ Sy1 & $1.1 \times 10^{-1}$ & B95 \\
HEAO-1 A2 & IRAS $12 \mu$ Sy2 & $3.1 \times 10^{-2}$ & B95 \\
\hline
\end{tabular}

TABLE 2. Cross-correlation signal at zero-lag $\left(W_{X g}\right)$ of galaxy catalogues with XRB data either from the HEAO-1 A2 experiment or the GINGA North Galactic Pole scans. References are M94: Miyaji et al (1994); C95: Carrera et al 1995; (B95): Barcons et al (1995).

are selected (see table 2). The interpretation of this signal requires to model the clustering of X-ray sources around the catalogued galaxies, which is indeed modulated by the bias parameter $b_{X}$. Using $b_{X}=1$, it is found that the local volume emissivity of optically selected galaxies amounts to $\sim 10^{39} h \mathrm{erg} \mathrm{s}^{-1} \mathrm{Mpc}^{-3}$ (Lahav et al 1993; Miyaji et al 1994; Carrera et al 1995), most of which is contributed by Seyfert galaxies and QSOs (Barcons et al 1995).

When this volume emissivity is extrapolated to higher redshifts, the fraction of the XRB intensity due to the precursors of the catalogued galaxies can be predicted (Lahav et al 1993). Carrera et al (1995) find that 10-30\% of the hard X-ray background might be produced by optically selected galaxies without exceeding the upper limits on the autocorrelation function of the XRB. This value is similar to the result of cross-correlation analyses of deep ROSAT X-ray images with deep optical images in the same fields (Almaini et al 1997).

A constraint on the bias parameter of X-ray sources from the CCF results can be derived by taking into account that a fraction $f \approx 2 / 3$ of the CCF signal arises from sources clustered with the catalogued galaxies. As that contribution scales linearly with $b_{X}$, the local volume emissivity scales $\propto \frac{3}{1+2 b_{X}}$. Since the AGN-only local emissivity is also $\sim 10^{39} \mathrm{erg} \mathrm{cm}^{-2} \mathrm{~s}^{-1}$, we can safely derive that $b_{X}<2$ as otherwise the total volume emissivity will be significantly less than the AGN emissivity.

\section{CLUSTERING OF X-RAY SELECTED SOURCES}

In the recent years large, complete samples of X-ray selected AGN have been built. This has allowed the direct measurement of the 3D spatial correlation function $\xi(r)$ for these objects and its comparison with the spatial correlation function of galaxies selected at other wavebands.

Carrera et al (1998) used two complete samples of X-ray selected AGN in pencil beam survey regions, spanning a wide redshift range $(0<z<2)$ to search for clustering signals and deriving its amplitude and redshift evolution. Clustering is 
found to be $99 \%$ significant at $z<1$. When the spatial correlation function is fitted to a standard power-law form $\xi(r)=(1+z)^{p}\left(\frac{r}{r_{0}}\right)^{-1.8}$ (for comoving $r$ ), it is seen that comoving or slower clustering evolution is excluded, and that even for stable or linear growth the values of $r_{0}$ permitted by the data are of the same order as the ones derived from clustering of IRAS galaxies. Carrera et al (1998) conclude that X-ray selected AGN are not significantly biased $0.7<b_{X}<2$.

Akylas, Plionis \& Georgantopoulos (1999) have used the ROS AT All Sky Survey sources to derive a local angular correlation function from which they estimate a somewhat higher correlation length $\left(r_{0} \sim 7-9 \mathrm{Mpc}\right)$, consistent with optically selected QSO clustering (La Franca et al 1998) and comoving clustering evolution. The obvious weakness of this method is that it is not based on $3 \mathrm{D}$ but $2 \mathrm{D}$ data.

\section{FLUCTUATIONS AND ANISOTROPIES IN THE XRB}

The method of auto-correlating the XRB intensity at various separations has been extensively used in an effort to detect small scale structure in the XRB attributable to source clustering (Barcons \& Fabian 1989, De Zotti et al 1990, Jahoda \& Mushotzky 1991, Carrera et al 1991, Carrera \& Barcons 1992, Carrera et al 1993, Chen et al 1994). These works produced a set of upper limits for the auto-correlation function of the XRB $W_{X X}(\theta)=\left\langle I_{X R B} I_{X R B}\right\rangle_{\theta} /\left\langle I_{X R B}\right\rangle^{2}-1$ on different angular scales (except for the Jahoda \& Mushotzky 1991 work, which claimed a detection at separations $\sim 10^{\circ}$ ) of the order of $10^{-3}-10^{-4}$ which constrained the clustering properties of the underlying source population (see, e.g., Fabian \& Barcons 1992).

Under the assumption of comoving clustering evolution, the sources of the XRB cannot be more strongly clustered than optically selected galaxies (see Carrera \& Barcons 1992), in which case $b_{X} \sim 1$. However, as explained above, Carrera et al (1998) found marginal evidence for faster clustering evolution in samples of X-ray selected AGN. This means that $b_{X}$ could be higher without violating the upper limits on the autocorrelation function, as the sources that produce the bulk of the $\mathrm{XRB}$ at high redshift could be very weakly clustered.

A further method employing the XRB angular variations has been to search for fluctuations in the XRB intensity distribution in excess of the ones expected from confusion noise produced by unresolved sources. These excess fluctuations should then be attributed to source clustering if all remaining noises (counting noise, systematics, etc.) could be removed. Studies of this kind have invariably lead to upper limits summarized in Table 3. What actually limits the sensitivity

of this method is the statistics: it scales as $N_{o b s}^{-1 / 2}$ where $N_{o b s}$ is the number of independent measurements of the XRB intensity that have been used to derive the excess fluctuations.

Excess fluctuations are related to the power spectrum of the density field of the Universe, weighted with the X-ray volume emissivity as a function of redshift (Barcons, Fabian \& Carrera 1998). The method is potentially very powerful as it reflects the clustering properties of the sources that produce the bulk of the XRB at redshifts $z>1$. 


\begin{tabular}{|l|c|c|l|}
\hline XRB data & Beam & $\left(\frac{\Delta I}{I}\right)_{\text {excess }}$ & Ref \\
\hline HEAO-1 A2 & $5^{\circ} \times 5^{\circ}$ & $<0.02$ & S83 \\
Ginga & $2^{\circ} \times 1^{\circ}$ & $<0.04$ & B97 \\
ROSAT & $\pi \times\left(2.5^{\prime}\right)^{2}$ & $<0.07$ & CFB97 \\
ROSAT & $\pi\left(10^{\prime 2}-5^{\prime 2}\right)$ & $<0.12$ & CFB97 \\
\hline
\end{tabular}

TABLE 3. Upper limits to excess fluctuations. References are S83: Shafer (1983); B97: Butcher et al (1997); CFB97: Carrera, Fabian \& Barcons (1997)

\begin{tabular}{|l|l|c|c|}
\hline Measurement & Reference & Scale $(\mathrm{Mpc})$ & $b\left(\Omega_{0}=1\right)$ \\
\hline X-ray cluster dipole & PK98 & $10-100$ & 4 \\
X-ray AGN dipole & MB90 & 1000 & $3-6$ \\
XRB-galaxy CCF & & $10-100$ & $<2$ \\
XRB dipole vs bulk motions & S99 & 1000 & $2-7$ \\
XRB multipoles vs bulk motions & T98 & $100-1000$ & $1-2$ \\
Clustering of distant AGN & C98 & $10-100$ & $1-2$ \\
Clustering of nearby AGN & APG99 & 100 & $2-3$ \\
\hline
\end{tabular}

TABLE 4. Bias parameters as inferred from various measurements. references are: PK98 Plionis \& Kolokotronis (1998); MB90: Miyaji \& Boldt (1990); S99: Scharf et al (1999); T98: Treyer et al (1998); C98: Carrera et al (1998); APG99: Akylas, Plionis \& Georgantopoulos (1999).

\section{THE BIAS PARAMETER OF X-RAY SOURCES}

Over the past sections we have discussed various approaches to detect and measure the clustering properties of X-ray sources. Table 4 summarizes the inferred bias parameter $b_{X}$ from these studies. Measurements are carried out with a variety of methods, correspond to different objects, are sensitive to different redshifts and also to different scales. Besides that, all dynamical estimates actually measure the combination $b_{X} \Omega_{0}^{-0.6}$.

Measurements of the correlation function are also affected by the cosmological parameters in the computation of the distances at significant redshifts, beyond the obvious linear dependence on $H_{0}$. If we live in an accelerating Universe, the Carrera et al (1998) correlation length would have to be scaled up by $30-50 \%$, resulting in a subsequent increase of almost a factor of 2 in the bias parameter. Given the uncertainties in the values of $q_{0}$ and $\Lambda$ (even for a flat Universe), the Carrera et al (1998) and Akylas et al (1999) results cannot be considered inconsistent.

As expected, clusters are a largely biased population $\left(b_{X} \sim 4\right)$ compared to AGN $\left(b_{X} \sim 1-2\right)$. The multipoles of the XRB are expected to be dominated by AGN, as 
these objects are the main sources of the XRB. The bias parameter derived from the $\mathrm{XRB}$ multipoles is consistently in agreement with the bias parameter derived from AGN clustering $\left(b_{X} \sim 1-2\right)$. The exception to this is the XRB dipole which implies a larger value of $b_{X}$. This could be partly due to a larger cluster contribution, as the lowest order multipoles are most sensitive to nearest (and brightest) sources, where the cluster contribution to the source counts $(\sim 10 \%$ on average in the deep extragalactic surveys) is $\sim 50 \%$ for the Piccinotti et al (1982) sample.

\section{FUTURE PROSPECTS}

$\mathrm{X}$-ray astronomy is now in a position to address cosmological studies. X-ray selected AGN which produce most of the X-rays in the Universe, appear to trace mass with a moderate bias parameter $b_{X} \sim 1-2$, but that has to be better defined as a function of scale and redshift. Chandra and XMM will carry out several deep 'pencil beam' surveys which, after subsequent identification of the serendipitous sources discovered, will define the redshift evolution of the AGN X-ray luminosity function at photon energies $>2 \mathrm{keV}$ and therefore the X-ray volume emissivity as a function of redshift. However, these surveys will not map sufficiently large areas of the sky which are necessary to trace the large-scale structure of the Universe at the redshifts where the XRB was produced.

The obvious way to go would be to survey very large areas of the sky (the whole sky even better) for X-ray sources, in order to have a most complete picture. Unless hard X-rays are produced at significantly lower redshifts than soft X-rays (which is doubtful in view of the $A S C A$ and BeppoSAX surveys), to reach $z \sim 1$ where a significant fraction of the X-ray emissivity in the Universe resides, these surveys will have to go at least down to $\sim 10^{-14} \mathrm{erg} \mathrm{cm}^{-2} \mathrm{~s}^{-1}$.

There is an alternative which is to perform high sensitivity observations of the XRB with a beam corresponding to the linear scale to be probed (Barcons, Fabian \& Carrera 1998). As the peak of the power spectrum of the density field of the Universe occurs at comoving wavenumbers $\sim 0.01-0.1 \mathrm{~h} \mathrm{Mpc}^{-1}$, for a standard geometry a $1^{\circ}$ resolution is well matched to this at $z \sim 1-3$. All-sky measurements of the XRB intensity on that angular scale with a precision of a few $\%$ could then be used to detect the excess fluctuations due to source clustering which are expected to be just below $1 \%$ in amplitude. Controlling all other possible sources of excess fluctuations well below that level requires a stable large-area detector (to reduce photon counting noise) and probably an X-ray monitor which images simultaneously the brightest sources in the field.

\section{ACKNOWLEDGEMENTS}

Partial financial support for this work was provided by the DGESIC under project PB95-0122. 


\section{REFERENCES}

Akylas, T., Plionis, M., Georgantopoulos, 1999, in: MPA/ESO conference on Evolution of Large Scale Structure, in the press

Almaini, O. et al 1997, MNRAS, 291, 372

Barcons, X., Fabian, A.C., 1989, MNRAS, 237, 119

Barcons, X., Franceschini, A., De Zotti, G., Danese, L., Miyaji, T., 1995, ApJ, 455, 480

Barcons, X., Fabian, A.C., Carrera, F.J., 1998, MNRAS, 293, 60

Boyle, B.J. et al, 1994, MNRAS, 271, 639

Boyle, B.J., Terlevich, R.J., 1996, MNRAS, 293, L49

Butcher, J.A. et al, 1997, MNRAS, 291, 437

Carrera, F.J. et al 1991, MNRAS, 249, 698

Carrera, F.J., Barcons, X., 1992, MNRAS, 257, 507

Carrera, F.J. et al 1993, MNRAS, 260, 376

Carrera, F.J. et al 1995, MNRAS, 275, 22

Carrera, F.J., Fabian, A.C., Barcons, X., 1997, MNRAS, 285, 820

Carrera, F.J. et al 1998, MNRAS, 299, 229

Chen, L.-W. et al, 1994, MNRAS, 266, 846

De Zotti, G., et al 1990, ApJ, 351, 22

Fixsen, D.J. et al, 1996, ApJ, 473, 576

Fabian, A.C., Barcons,X., 1992, ARAA, 30, 429

Fry, J.N., 1996, ApJ, 461, L65

Iwan, D. et al, 1982, ApJ, 260, 111

Jahoda, K., Mushotzky, R.F., 1989, ApJ, 346, 638

Kolokotronis, V., Plionis, M., Coles, P., Borgani, S., 1998, MNRAS, 295, 19

La Franca, F., Andreani, P., Cristiani, S., 1998, ApJ, 497, 529

Lahav, O. et al 1993, Nat, 364, 693

Lahav, O., Piran, T., Treyer, M.A., 1997, MNRAS, 284, 499

Madau, P. et al 1996, MNRAS, 283, 1388

Miyaji, T., Boldt, E., 1990, ApJ, 353, L3

Miyaji, T., 1994, PhD Thesis, Univ of Maryland

Miyaji, T., Hasinger, G., Schmidt, M., 1999, A\&A, in press

Mushotzky, R., Jahoda, K., 1992, in: The X-ray background, Barcons, X., Fabian, A.C. eds, CUP

Page, M.J. et al 1996, MNRAS, 281, 597

Persic, M. et al, 1990, ApJ, 364, 1

Piccinotti, G. et al, 1982, ApJ, 253, 485

Plionis, M., Kolokotronis, V., 1998, ApJ, 500, 1

Plionis, M., Georgantopoulos, I., 1999, MNRAS, in the press

Scharf, C., Jahoda, K., Treyer, M., Lahav, O., Boldt, E., Piran, T., 1999, ApJ, submitted

Shafer, R.A., 1983, PhD thesis, Univ of Maryland

Shafer, R.A., Fabian, A.C., 1983, in: IAU Symposium 104, Early evolution of the Universe and its present structure, Reidel, p. 333

Treyer, M., Scharf, C., Lahav, O., Jahoda, K., Boldt, E., Piran, T., 1998, ApJ, 509, 531

Warwick, R.S., Pye, J.P., Fabian, A.C., 1980 , MNRAS, 190, 243 